\title{
GENERAL INDEX TO VOLUME 52 (1995)
}

Aeschynanthus arctocalyx, a new species from the Philippines, 343

Aeschynanthus arctocalyx Mendum \& Madulid, 343

confertus Griffith, 219

kingii C.B. Clarke, 219

levipes C.B. Clarke, 220

oxychlamys Mendum, 362

pullei (Schltr.) P. Royen, 362

Alectoria californica (Tuck.) Merrill, 108 nigricans (Ach.) Nyl., 108 sarmentosa (Ach.) Ach., 108 vancouverensis (Gyelnik) Gyelnik ex

Brodo \& D. Hawksw., 108

Anaptychia ciliaris Ach., 124 erinacea (Ach.) Trevisan, 110

Anchusa negevensis Danin, 333

Anomalluma Plowes, 202 dodsoniana (Lavranos) Plowes, 202

ANOZIE, V.C., A new species of Terminalia from Nigeria, 347

Anthodendron Reichenbach, 11

ARGENT, G.C.G., New combinations and synonymy in some rhododendrons of section Vireya, 363

Arthothelium ilicinum (Taylor) P. James, 108

Astrosporina aequalis Horak, 353 asterospora (Quél.) Rea, 353

corneri Horak, 355

\section{Azalea L.}

subgen. Pentanthera (G. Don) Koch, 11 subgen. Rhodora L., 26

subgen. Sciadorhodion (Rehder \&

Wilson) Copeland, 13

albrechtii (Maxim.) Kuntze, 22

canadensis (L.) Kuntze, 22

nipponica (Matsum.) Copeland, 24

pentaphylla (Maxim.) Copeland, 19

quinquefolia (Bisset \& S. Moore)

Olmstead, Colville \& Kelsey, 16

schlippenbachii (Maxim.) Kuntze, 15

vaseyi (A. Gray) Rehder, 27

Bacidia sp., 108, 123

Baeomyces acicularis Ach., 114

Basidiomycetes, Incompatibility in, 71

BATEMAN, R.M. \& DENHOLM, I., The Hebridean Marsh-Orchid:
Nomenclatural and conceptual

clarification of a biological enigma, 55

Biltia Small, 26

vaseyi (A. Gray) Small, 27

BOOK REVIEWS

The Common Ground of Wild and Cultivated Plants, edited by A.R. Perry and R.G. Ellis, 91

C.P. Thunberg's Drawings of Japanese Plants, edited by Y. Kimura and V.P. Leonov, 238

The Genera of the Umbelliferae, by M.G. Pimenov and M.V. Leonov, 92

An Illustrated Flora of North Cyprus, by D.E. Viney, 93

Scarce Plants in Britain, compiled and edited by A. Stewart, D.A. Pearman and C.D. Preston, 237

Siebold's Florilegium of Japanese Plants, edited by Y. Kimura and V.I. Grubov, 238

Borrera ceruchis (Ach.) Ach., 114 erinaceus Ach., 110

BRUYNS, P.V. \& MEVE, U., The generic position of Caralluma dodsoniana, 195

Bryoria bicolor (Ehrh.) Brodo \& D. Hawksw., 108 capillaris (Ach.) Brodo \& D. Hawksw., 108

Bunodophoron australe (Laurer) A. Massal, 102

patagonicum (Dodge) Wedin, 102

BURTT, B.L., See HILLIARD

Caloplaca flavorubescens (Huds.)

Laundon, 108

Caralluma dodsoniana, The generic position of, 195

Caralluma dodsoniana Lavranos, 202

Catillaria sp., 108

Cavernularia lophyrea (Ach.) Degel., 108

Cenomyce acicularis (Ach.) Ach., 115

Cetraria californica Tuck., 108 islandica subsp. crispiformis (Räsänen) Kärnefelt, 109 lacunosa Ach., 116

f. cavernosa (Menzies) Merrill, 117

f. cavernosus (Menzies in Ach.)

Merrill, 117 
Cetraria orbata (Nyl.) Fink, 109

Chaenotheca brunneola (Ach.) Müll.

Arg., 102

Chlorodictyon foliosum J. Agardh, 120

Chrysothrix candelaris (L.) Laundon, 103

Cladia aggregata (Sw.) Nyl., 103

Cladina confusa (R. Sant.) Follman \&

Ahti, 129

portentosa subsp. pacifica (Ahti) Ahti, 109

pycnoclada (Pers.) Leighton, 103

rangiferina (L.) Nyl., 109

Cladonia acicularis (Ach.) Fr., 115

amaurocraea (Flörke) Schaerer, 109

bacillaris Nyl., 103

bellidiflora (Ach.) Schaerer, 109

cervicornis subsp. verticillata (Hoffm.)

Ahti, 101, 109

crispata (Ach.) Flotow, 109

cristatella Tuck., 101

furcata (Huds.) Schrader, 109

gracilis subsp. elongata (Jacq.) Vainio, 103

macilenta Hoffm., 128

maxima (Asah.) Ahti, 101, 109

subfurcata (Nyl.) Arnold, 109

transcendens (Vainio) Vainio, 109

uncialis (L.) Weber ex Wiggers, 109

verruculosa (Vainio) Ahti, 109

Coccotrema pocillarium (Cummings)

Brodo, 109

Coelocaulon aculeatum (Schreber) Link, 110

californicum (Tuck.) Howe, 108

Coenogonium implexum Nyl., 126

Collema conglomeratum var.

crassiusculum (Malme) Degelius, 130

menziesii (Ach.) Ach., 103

rugosum Krempelh., 123

subconveniens Nyl., 124, 126

COPPINS, B.J. \& KONDRATYUK,

S.Y., Stygiomyces and Pseudonitschkia:

Two new genera of lichenicolous fungi, 229

Cornicularia californica (Tuck.) Du Rietz, 108

Crocodia obvoluta (Ach.) Trevisan, 104

CRONK, Q.C.B., A new species and hybrid in the St Helena endemic genus

Trochetiopsis, 205
Cyanistica anthraspis (Ach.) Gyelnik., 118 obvoluta (Ach.) Dodge, 104

Dactylorhiza majalis (Reichenbach f.)

P.F. Hunt \& Summerhayes subsp.

occidentalis (Pugsley) Soó var.

ebudensis Wiefelspütz ex R.M. Bateman

\& Denholm, 57

subsp. occidentalis var. ebudensis

Wiefelspütz, 57

var. scotica (Nelson) R.M. Bateman

\& Denholm, 57

subsp. scotica Nelson, 57

DANIN, A., A new Anchusa from Israel, 333

DENHOLM, I., See BATEMAN

Dermatocarpon luridum (With.)

Laundon, 101

Desmazieria ceruchis (Ach.) Trevisan, 114

homalea (Ach.) Mont., 114

Dictyonema glabratum (Sprengel)

D. Hawksw., 130

Didymocarpus and Lysionotus, Notes on, 215

Didymocarpus aureoglandulosus C.B.

Clarke, 215

curvicapsa Hilliard, 217

obtusa auct. non [Wall. ex] R. Br.; C.B.

Clarke, 218

pinetorum Kurz, 215

rodgeri W.W. Smith, 215

var. siamensis W.W. Smith, 215

subalternans $\mathrm{R}$. Br. var. curvicapsa C.B.

Clarke, 217

var. curvicapsularis C.B. Clarke, 217 triplotrichus Hilliard, 218

Dimerella lutea (Dickson) Trevisan, 110

Diploicia canescens (Dickson) Massal., 101

Dirinaria picta (Sw.) Clem. \& Shear, 123

Distichochlamys, a new genus from

Vietnam, 65

Distichochlamys M.F. Newman, 65

citrea M.F. Newman, 65

Dufourea simplex Taylor, 121

Erioderma pulchrum Müll. Arg., 129

The extra-European lichen collections of Archibald Menzies MD, FLS

(1754-1842), 95 
GALLOWAY, D.J., The extra-European lichen collections of Archibald Menzies MD, FLS (1754-1842), 95

The generic position of Caralluma dodsoniana, 195

Graphina sp., 123

Graphis sp., 110

Graphis scripta (L.) Ach., 101

The Hebridean Marsh-Orchid:

Nomenclatural and conceptual clarification of a biological enigma, 55

Hemiboea himalayensis Lévl., 221

Heterodermia comosa (Eschw.) Follm. \& Redon, 129

erinacea (Ach.) W. Weber, 110

lepidota Swinscow \& Krog, 124

leucomelos (L.) Poelt, 124, 129-30

magellanica (Zahlbr.) Swinscow \&

Krog, 124

podocarpa (Bel.) Awasthi, 129

speciosa (Wulfen) Trevisan, 124

HILLIARD, O.M.

New names in Selago, 361

Pseudoselago, a new segregate from Selago, 243

HILLIARD, O.M. \& BURTT, B.L., OId

World Gesneriaceae. IV. Notes on

Didymocarpus and Lysionotus, 215

Hochenwartia Crantz, 26

canadensis (L.) Crantz, 30

Hypogymnia duplicata (Ach.) Rass., 110 enteromorpha (Ach.) Nyl., 110

lophyrea (Ach.) Krog, 108

Icmadophila ericetorum (L.) Zahlbr., 101, 110

IKEDA, H. \& OHBA, H., A new species of Potentilla sect. Leptostylae from central Burma, 225

Imbricaria enteromorpha (Ach.) Jatta, 110

Incompatibility in basidiomycetes: The heterogenic Pentax, 71

Inocybe in Peninsular Malaysia, 351

Inocybe aequalis (Horak) Turnbull \&

Watling, 353

asterospora Quél., 352, 353

aurantiocystidiata Turnbull \& Watling, 352,355

corneri (Horak) Garrido, 353, 355 cutifracta Petch, 352, 356

fuscospinulosa Corner \& Horak, 352, 356

lutea Kobayasi \& Hongo, 353, 357

palaeotropica Turnbull \& Watling, 352, 357

sphaerospora Kobayasi, 352, 358

umbrina Massee, 357

JUDD, W.S. \& KRON, K.A., A revision of Rhododendron VI. Subgenus

Pentanthera (Sections Sciadorhodion, Rhodora and Viscidula), 1

KEMP, R.F.O., Incompatibility in basidiomycetes: The heterogenic Pentax, 71

KLJUYKOV, E.V., See PIMENOV

KONDRATYUK, S.Y., See COPPINS

Korshinskya extended westwards, 337

Korshinskya assyriaca (Freyn. \& Bornm.)

Pimenov \& Kljuykov, 339, 342

bupleuroides Korovin, 342

kopetdaghensis (Korov) Pimenov \&

Kljuykov, 342

olgae (Regel \& Schmalh.) Lipsky, 342

rapifera (Gilli) Podlech \& Rech. f., 342

KRON, K.A., See JUDD

Lasallia papulosa var. rubiginosa (Pers.)

Llano, 124

Lecanactis concordans (Nyl.) Zahlbr., 123

Lecanora pacifica Tuck., 110

Lecidea sp., 110

Lecidella elaeochroma (Ach.) M. Choisy, 103

Leifidium tenerum (Laurer) Wedin, 126

Lepraria nivalis Laundon, 110

Leptogium sp., 123, 129

austroamericanum (Malme) Dodge, 124,129

azureum (Sw.) Mont., 124, 129

brebissonii Mont., 126

coralloideum (Meyen \& Flotow)

Vainio, 124

corniculatum (Hoffm.) Minks, 111, 124

corticola (Taylor) Tuck., 123

cyanescens (Rabenh.) Körber, 123, 129

menziesii (Ach.) Mont., 103 
moloccanum (Pers.) Vainio, 129

palmatum (Hudson) Mont., 125

Letharia vulpina (L.) Hue, 112

Lethariella canariensis (Ach.) Krog., 125

Lichen collections of Archibald Menzies, 95

Lichen bullatus Menzies, 103

cavernosus Menzies, 116

cincinnatus Sm., 103

duplicatus $\mathrm{Sm} ., 110$

erinaceus $\mathrm{Sm} ., 110$

intestinalis $\mathrm{Sm} ., 110$

lacunosus Menzies, 114

menziesii Sm., 103

obvolutus Sm., 104

planus Menzies, 114

reticulatus Noehden, 119

Lichenicolous fungi, Two new genera of, 229

Lithocaulon P.R.O. Bally, 202

Lobaria crenulata (Hook.) Trevisan, 129 orygmaea (Ach.) Hellbom, 104

pulmonaria (L.) Hoffm., 112

querzicans Michaux, 101

Loxospora ochrophaea (Tuck.) R.C.

Harris, 101

Lysionotus and Didymocarpus, Notes on, 215

Lysionotus angustisepalus W.T. Wang, 220

atropurpureus Hara, 219

confertus C.B. Clarke, 219

gracilipes C.E.C. Fischer, 220

himalayensis (Lévl.) W.T. Wang, 221

kingii (C.B. Clarke) Hilliard, 219

levipes (C.B. Clarke) B.L. Burtt, 220

pubescens C.B. Clarke, 220

serratus D. Don, 221

var. pterocaulis W.T. Wang, 224

ternifolius Wall., 221

wardii W.W. Smith, 220

\section{MADULID, D., See MENDUM}

Megalospora sulphurata Meyen, 125

Melhania melanoxylon $\mathrm{R}$. Br. ex W.T.

Aiton, 207

MENDUM, M., Aeschynanthus oxychlamys Mendum nom. nov.

(Gesneriaceae), 362

MENDUM, M. \& MADULID, D., Aeschynanthus arctocalyx, a new species from the Philippines, 343
Menegazzia cincinnata (Ach.) Bitter, 103

Menzies, Archibald, 95

MEVE, U., See BRUYNS

Nephroma antarcticum (Jacq.) Nyl., 103

bellum (Sprengel) Tuck., 113

cellulosum (Ach.) Ach., 103

laevigatum Ach., 114

resupinatum (L.) Ach., 114

Nephromium cellulosum (Ach.) Nyl., 103

Neuropogon aurantiaco-ater (Jacq.)

Lamb, 103

A new Anchusa from Israel, 333

New combinations and synonymy in some rhododendrons of section Vireya, 363

New names in Selago, 361

A new species of Potentilla sect.

Leptostylae from central Burma, 225

A new species of Terminalia from Nigeria, 347

NEWMAN, M.F., Distichochlamys, a new genus from Vietnam, 65

Niebla ceruchis (Ach.) Rundel \& Bowler, 114 combeoides (Nyl.) Rundel \& Bowler, 114

homalea (Ach.) Rundel \& Bowler, 114

Notes on Didymocarpus and Lysionotus, 215

OHBA, H., See IKEDA

Old World Gesneriaceae. IV. Notes on

Didymocarpus and Lysionotus, 215

OLIVEIRA-FILHO, A.T. \& RATTER, J.A., A study of the origin of central

Brazilian forests by the analysis of plant species distribution patterns, 141

Opegrapha atra Pers., 125

Opisteria cellulosa (Ach.) Vainio, 103

Oxychlamys pullei Schltr., 362

Pannaria brisbanensis C. Knight, 125

lurida (Mont.) Nyl., 125

pezizoides (G.H. Weber) Trevisan, 104, 114,130

rubiginosa (Thunb.) Delise, 129

Parmelia ceruchis Ach., 114

cincinnata Ach., 103

cribellata Taylor, 108 
duplicata Ach., 110

enteromorpha Ach., 110

fertilis Müll. Arg., 101

lophyrea Ach., 108

menziesii Ach., 103

obvoluta Ach., 104

physodes var. enteromorpha (Ach.)

Tuck., 110

saxatilis (L.) Ach., 101

sulcata Taylor, 101

Parmeliella brisbanensis (C. Knight) P.M.

Jørg. \& D.J. Galloway, 123

mariana (Fries) P.M. Jørg. \&

D.J. Galloway, 123, 125, 129

Parmelina galbina (Ach.) Hale, 101

Parmotrema arnoldii (Du Rietz) Hale, 114

dilatatum (Vainio) Hale, 114

perforatum (Jacq.) Massal., 114

Peltidea cellulosa Ach., 103

Peltigera membranacea (Ach.) Nyl., 114 venosa (L.) Hoffm., 114

Pentapetes melanoxylon Sol. ex Sims, 207

Pertusaria sp., 104

Phaeophyscia endococcina (Körber)

Moberg, 125

Phyllopodium rudolphii Hiern., 316

Phyllopsora sp., 114 corallina (Eschw.) Müll. Arg., 125

Physcia ciliaris var. erinacea (Ach.) Nyl., 110

erinacea (Ach.) Tuck., 110

leucomela var. erinaceus (Ach.) Nyl., 110

Physma byrsaeum (Ach.) Tuck., 125, 128-9

Pilophorus acicularis (Ach.) Th. Fr., 114 cereolus var. acicularis (Ach.) Tuck.,

115

PIMENOV, M.G. \& KLJUYKOV, E.V., Korshinskya extended westwards, 337

Platismatia glauca (L.) Culb. \& C. Culb., 116

herrei (Imsh.) Culb. \& C. Culb., 116 lacunosa (Ach.) Culb. \& C. Culb., 116 stenophylla (Tuck.) Culb. \& C. Culb., 118

Platysma lacunosum (Ach.) Nyl., 117

Potentilla sect. Leptostylae from central

Burma, 225
Potentilla montisvictoriae H. Ikeda \&

H. Ohba, 225

Pseudocyphellaria anomala Brodo \&

Ahti, 118

anthraspis (Ach.) H. Magn., 118

aurata (Ach.) Vainio, 129-30

berberina (G. Forster) D. Galloway \&

P. James, 104

billardieri (Delise) Räsänen, 126

cinnamomea (A. Rich.) Vainio, 126

clathrata (De Not.) Malme, 130

coronata (Müll. Arg.) Malme, 126

crocata (L.) Vainio, 118

dubia Du Rietz, 104

faveolata (Delise) Malme, 126

freycinetii (Delise) Malme, 104

gilva (Ach.) Malme, 125

hirsuta (Mont.) Malme, 104

lividofusca (Krempelh.) D. Galloway \&

P. James, 126

multifida (Nyl.) D. Galloway \&

P. James, 126

obvoluta (Ach.) Malme, 104

orygmaea (Ach.) Malme, 104

punctillaris (Müll. Arg.)

D.J. Galloway, 129

Pseudolithos P.R.O. Bally, 202

dodsonianus (Lavranos) Bruyns \&

Meve, 202

Pseudonitschkia Coppins \& Kondratyuk, 232

parmotrematis Coppins \& Kondratyuk, 232

Pseudoselago, a new segregate from Selago, 243

Pseudoselago arguta (E. Mey.) Hilliard, 313

ascendens (E. Mey.) Hilliard, 270

bella Hilliard, 326

burmannii (Choisy) Hilliard, 310

caerulescens Hilliard, 279

candida Hilliard, 297

densifolia (Hochst.) Hilliard, 306

diplotricha Hilliard, 324

gracilis Hilliard, 289

guttata (E. Mey.) Hilliard, 267

humilis (Rolfe) Hilliard, 316

langebergensis Hilliard, 275

outeniquensis Hilliard, 282

parvifolia Hilliard, 294 
peninsulae Hilliard, 265

prolixa Hilliard, 322

prostrata Hilliard, 328

pulchra Hilliard, 250

quadrangularis (Choisy) Hilliard, 258

rapunculoides (L.) Hilliard, 303

recurvifolia Hilliard, 256

serrata (P.J. Bergius) Hilliard, 252

similis Hilliard, 318

sp., 309

spuria (L.) Hilliard, 284

subglabra Hilliard, 300

verbenacea (L.f.) Hilliard, 273

violacea Hilliard, 261

Psoroma hypnorum (Vahl) S.F. Gray, 104

lepromum (Nyl.) Räsänen, 105

pholidotoides (Nyl.) Trevisan, 105

Pyrenula sp., 106

Pyxine petricola Nyl., 129

Ramalina arabum (Dill. ex Ach.) Meyen \& Flotow, 130

celastri (Sprengel) Krog \& Swinscow, 125,129

subsp. ovalis (J.D. Hook. \& Taylor)

Stevens, 129

complanata (Sw.) Ach., 118

dilacerata (Hoffm.) Hoffm., 118

homalea Ach., 114

leptocarpha Tuck., 118

menziesii Taylor, 118

reticulata Krempelh., 119

retiformis Menzies ex Tuck., 120

sp., 121, 129

tayloriana Zahlbr., 129

thrausta (Ach.) Nyl., 121

usnea (L.) R. Howe, 121

RATTER, J.A., See OLIVEIRA-FILHO

A revision of Rhododendron VI. Subgenus

Pentanthera (Sections Sciadorhodion,

Rhodora and Viscidula), 1

Rhododendron, A revision of, 1

Rhododendron

sect. Azalea Planchon, 13

sect. Pentanthera G. Don, 11

sect. Pentanthera G. Don, 13

sect. Rhodora (L.) G. Don, 1, 26

sect. Sciadorhodion Rehder \& Wilson, 1,13 sect. Viscidula Matsum. \& Nakai, 1, 24

subgen. Anthodendron, 11

subgen. Pentanthera (G. Don)

Poyarkova, 1, 11

subgen. Pseudanthodendron Sleumer, 11

Anthodendron (Reichenbach) Endl., 11

albrechtii Maxim., 22, 36

borneense (J.J. Sm.) Argent, A.L.

Lamb \& Phillipps

subsp. angustissimum (Sleumer)

Argent, 363

subsp. brookeanum

var. cockburnii (Argent, A.L.

Lamb \& Phillipps) Argent, 364

var. kinabaluense (Argent, A.L.

Lamb \& Phillipps) Argent, 363

var. moultonii (Ridl.) Argent, 364

subsp. gracile (Lindley) Argent, 364

var. gracile Hensl., 364

canadense (L.) Torrey, 30, 37

f. albiflorum (E.L. Rand \& Redf.)

Rehder, 30

f. album Voss, 30

f. viridifolium Fernald, 30

flavum G. Don, 11

gracile Low ex Lindl., 364

javanicum (Blume) Bennett

subsp. brookeanum var. kinabaluense

Argent, Lamb \& Phillipps, 363

subsp. cockburnii Argent, Lamb \&

Phillipps, 364

subsp. moultonii (Ridl.) Argent, 364

subsp. schadenbergii Argent, 364

var. schadenbergii (Warb.) Sleumer, 364

kinabaluense Merr., 365

konori Becc. var. phaeopeplum

(Sleumer) Argent, 364

leptanthum F. Muell. var. warianum

(Schltr.) Argent, 365

luteum Sweet, 11

moultonii Ridl., 364

nikoense (Komatsu) Nakai, 20

nipponicum Matsum., 24, 37

pentaphyllum Maxim., 19, 36

var. nikoense Komatsu, 20

var. shikokianum T. Yamazaki, 20

phaeopeplum Sleumer, 364

quadrasianum Vidal var. angustissimum

Sleumer, 363 
quinquefolium Bisset \& S. Moore, 13,

16,35

f. speciosum N. Yonezawa, 17

var. roseum Rehder, 20

rhodora J.F. Gmelin, 30

f. albiflora E.L. Rand \& Redf., 30 rugosum Low ex Hook. f. var. laeve

Argent, A.L. Lamb \& Phillipps, 365

schadenbergii Warburg, 364

schlippenbachii Maxim., 15, 35

vaseyi $A$. Gray, 27, 37

f. album (Bean) Rehder, 27

var. album Bean, 27

warianum Schltr., 365

canadensis L., 30

Rhodora L., 26

Rimelia cetrata (Ach.) Hale \& Fletcher,

121

reticulata (Taylor) Hale \& Fletcher, 125,130

Roccella arnoldii Vainio, 125

Scaligeria assyriaca Freyn. \& Bornm., 339

Sciadorhodion, 1

Selago acutibractea Hilliard, 361

arguta $\mathrm{E}$. Mey., 313

ascendens auct., 261

ascendens $\mathrm{E}$. Mey., 270

burmannii Choisy, 310

coccinea L., 303

densifolia Hochst., 306

dentata Poir., 284

elata Rolfe, 361

fasciculata L., 252

var. glabra E. Mey. subvar. $b, 256$

var. hirta E. Mey., 258

subvar. $b, 265$

var. $\beta$ lanceolata Walp., 303

fulvomaculata Link, 284

guttata E. Mey., 267

heterophylla auct., 318

heterophylla Rolfe, 318

heterophylla Thunb., 284

hirta auct., 258

hirta L.f., 260

humilis Rolfe, 316

hyssopifolia auct., 361

hyssopifolia E. Mey. subsp. retrotricha

Hilliard, 361

incisa Hochst., 270 lanceolata Choisy, 303

lanceolata Choisy p.p., 310

pallida Salisb., 284

pentheri Gand., 252

procera Hilliard, 361

pulchella Salisb., 303

quadrangularis auct., 265

quadrangularis Choisy, 258

ramulosa Link, 275

rapunculoides $\mathrm{L}$., 303

var. $\beta$ densifolia (Hochst.) Choisy, 306

rudolphii (Hiern.) Levyns, 316

serrata P.J. Bergius, 252

spuria $\mathrm{L} ., 284$

tarachodes Hilliard, 361

teretifolia Link, 303

verbenacea L.f., 273

zuluensis Hilliard, 361

Siphula ceratites (Wahlenb.) Fr., 121

var. simplex (Taylor) Müll. Arg., 121

simplex (Taylor) Nyl., 121

verrucigera (J.F. Gmelin) R. Sant., 125

Solorina spongiosa (Ach.) Anzi, 121

Sphaerophorus melanocarpus (Sw.) DC., 121

Squamarina cartilaginea (With.) P.

James, 125

Stereocaulon aciculare (Ach.) Tuck., 115

implexum Th. Fr., 106

ramulosum (Sw.) Rauschel, 106, 126

vesuvianum Pers., 106

Sticta anthraspis Ach., 118

caulescens De Not., 106

damaecornis (Sw.) Ach., 129

filix (Sw.) Nyl., 127

fuliginosa (Hoffm.) Ach., 106, 121

gaudichaldia Delise, 106

latifrons A. Rich., 127

obvoluta (Ach.) Ach., 104

orygmaea Ach., 104

samoana (Müll. Arg.) Zahlbr., 128

subcaperata (Nyl.) Nyl., 128

Stygiomyces and Pseudonitschkia: Two

new genera of lichenicolous fungi, 229

Stygiomyces Coppins \& Kondratyuk, 229 galliformis Coppins \& Kondratyuk, 229

A study of the origin of central Brazilian forests by the analysis of plant species distribution patterns, 141 
Teloschistes chrysophthalmus (L.) Th.

Fr., 121

exilis (Michaux) Vainio, 125

flavicans (Sw.) Ach., 129-30

Terminalia pennyana Anozie, 347

Thamnolia subuliformis (Ehrh.) Culb., 121

Thelomma mammosum (Hepp in

Hartung) Massal., 121

Thelotrema lepadinum (Ach.) Ach., 107

Trochetiopsis ebenus Cronk, 210 melanoxylon (Sol. ex Sims) Marais, 207

Trochetiopsis $\times$ benjamini Cronk, 211

TURNBULL, E., Inocybe in Peninsular Malaysia, 351

Umbilicaria vellea (L.) Ach., 121

Usnea cavernosa Tuck., 122

ceratina Ach., 122

ceruchis (Ach.) Mont., 114

fulvoreagens (Räsänen) Räsänen, 122 intercalaris Krempelh., 129

longissima Ach., 122

lutea Motyka, 129

nidifica Taylor, 128

pruinosa Motyka, 129

strigosa (Ach.) A. Eaton, 101

subflorida (Zahlbr.) Mot., 125

subfloridana Stirton, 122

trichodea Ach., 101

trichodeoides Mot., 128

Viscidula, 1

Vulpicida canadensis (Räsänen) Mattson \& Lai, 122

Xanthomaculina hottentota (Ach.) Hale, 125

Xanthoria candelaria (L.) Th. Fr., 123 elegans (Link) Th. Fr., 101

parietina (L.) Th. Fr., 125 